To appear in Integral Transforms and Special Functions

Vol. 00, No. 00, Month 201X, 1-16

\title{
On linear spectral transformations and the Laguerre-Hahn class
}

\author{
K. Castillo ${ }^{a}$ and M.N. Rebocho ${ }^{b *}$ \\ ${ }^{a}$ CMUC, Department of Mathematics, University of Coimbra, Apartado 3008, EC Santa Cruz, \\ 3001-501 Coimbra, Portugal. \\ ${ }^{b}$ Departmento de Matemática, Universidade da Beira Interior, 6201-001 Covilhã, Portugal; \\ CMUC, Apartado 3008, EC Santa Cruz, 3001-501 Coimbra, Portugal. \\ (Received 00 Month 201X; final version received 00 Month 201X)
}

\begin{abstract}
We study the Christoffel and Geronimus transformations for Laguerre-Hahn orthogonal polynomials on the real line. It is analysed the modification on the corresponding differencedifferential equations that characterize the systems of orthogonal polynomials and the consequences for the three-term recurrence relation coefficients.
\end{abstract}

Keywords: Orthogonal polynomials; Laguerre-Hahn class; Riccati differential equation; Christoffel transformation; Geronimus transformation.

AMS Subject Classification: 33C47, 42C05.

\section{Introduction}

The study of the Christoffel and Geronimus transformations, members of the so-called canonical linear spectral transformations, is a widely known theme in the literature of orthogonal polynomials and special functions. From the historical point of view, the analysis of Christoffel and Geronimus transformations puts emphasis on modifications of weights supported on subsets of the real line under the multiplication and polynomial division with the addiction of a mass point, respectively. The seminal references are traced back to [1-5].

When the original weight is a member of the semi-classical class, that is, the logderivative is a rational function, there are well-known applications, e.g., in quantum mechanics, and in the theory of integrable systems (see, for instance, [6-10]). A common topic of research concerns the formulae expressing the new orthogonal polynomials in terms of the original system. Basic structures to be analysed are the consequences for the recurrence relation coefficients and the spectral derivatives of the corresponding system of orthogonal polynomials. Let us note that, when dealing with semi-classical families supported on the real line, the spectral derivatives are, essentially, systems of differencedifferential equations involving two consecutive elements of the sequence of orthogonal polynomials, $\left\{P_{n}\right\}_{n \geq 0}$, say,

$$
A P_{n}^{\prime}=M_{n} P_{n}+N_{n} P_{n-1}
$$

\footnotetext{
* Corresponding author. Email: mneves@ubi.pt
} 
where $A, M_{n}, N_{n}$ are polynomials. Equations such as (1) have been extensively studied from several points of view, allowing classifications and characterizations [11, 12].

In the present paper we study the action of the Christoffel and Geronimus transformations on Laguerre-Hahn families of orthogonal polynomials. Such sequences of orthogonal polynomials generalize the semi-classical families. Indeed, the Laguerre-Hahn class includes, as subclass, the semi-classical families as well as some of their transformations, including the linear spectral ones [13-15].

The main motivation for the present work relies on studies concerning polynomial and rational modification of weights in the semi-classical class, [8] and [10, Sec. 3].

A fundamental tool in our study are the systems of difference-differential equations involving the Laguerre-Hahn orthogonal polynomials and their associated, $\left\{P_{n}^{(1)}\right\}_{n \geq 0}$ (cf. Section 2),

$$
A \Psi_{n}^{\prime}=\mathcal{M}_{n} \Psi_{n}+\mathcal{N}_{n} \Psi_{n-1}, \quad n \geq 0, \quad \Psi_{n}=\left[\begin{array}{ll}
P_{n+1} & P_{n}^{(1)}
\end{array}\right]^{T},
$$

where $A$ is a polynomial and $\mathcal{M}_{n}, \mathcal{N}_{n}$ are $2 \times 2$ matrices with polynomial entries [16]. We study the modifications of systems of type (2) under the general framework of Christoffel and Geroninums transformations of linear functionals [15, 17, 18]. The main goal is to study the derivatives of the system (2) under such modifications and, as a consequence, to analyse the corresponding changes on the recurrence relation coefficients of the orthogonal polynomials. Our main results give the new matrices in (2) for the modified system in terms of the original one (cf. Section 4). Also, in the semi-classical case, we give the modified transfer matrices in terms of the original ones.

The reminder of the paper is organized as follows. In Section 2 we give the basic results on Laguerre-Hahn orthogonal polynomials and we introduce notation to be used in the sequel. In Section 3 we deduce formulae expressing the new matrices $\Psi_{n}$ in terms of the original ones (see Theorems 3.1, 3.3, and 3.5), from which the recurrence relation coefficients for the modified orthogonal polynomials are recovered (see Corollaries 3.2 and 3.4). In Section 4 we deduce the modified matrices of systems of type (2) under the Christoffel and Geronimus transformations for the generic Laguerre-Hahn class (see Theorem 4.1), together with the analysis of the transformations in the semi-classical class (see Theorem 4.2). Examples, illustrating the main results, are given in Sub-Section 4.2.

\section{Preliminary results and notations}

Let $\mathbb{P}=\operatorname{span}\left\{x^{k}: k \in \mathbb{N}_{0}\right\}$ be the linear space of polynomials with complex coefficients, and let $\mathbb{P}^{*}$ be its algebraic dual space. We will denote by $\langle u, f\rangle$ the action of $u \in \mathbb{P}^{*}$ on $f \in \mathbb{P}$. Given the moments of $u, u_{n}=\left\langle u, x^{n}\right\rangle, n \geq 0$, where we take $u_{0}=1$, the principal minors of the corresponding Hankel matrix are defined by $H_{n}=\operatorname{det}\left(u_{i+j}\right)_{i, j=0}^{n}$, where, by convention, $H_{-1}=1$. The functional $u$ is said to be quasi-definite (respect., positive-definite) if $H_{n} \neq 0$ (respect., $H_{n}>0$ ), for all $n \geq 0$.

Let $u \in \mathbb{P}^{*}$ and let $\left\{P_{n}\right\}_{n \geq 0}$ be a sequence of polynomials such that $\operatorname{deg}\left(P_{n}\right)=n$. The basis $\left\{P_{n}\right\}_{n \geq 0}$ is said to be a sequence of orthogonal polynomials with respect to $u$ if

$$
\left\langle u, P_{n} P_{m}\right\rangle=h_{n} \delta_{n, m}, h_{n} \neq 0, \quad n, m \geq 0 .
$$

Throughout the paper we shall take each $P_{n}$ monic, that is, $P_{n}(x)=x^{n}+$ lower degree terms, and we will denote $\left\{P_{n}\right\}_{n \geq 0}$ by SMOP.

The equivalence between the quasi-definiteness of $u$ and the existence of a SMOP with respect to $u$ is well-known in the literature of orthogonal polynomials $[19,20]$. 
Furthermore, if $u$ is positive-definite, then it has an integral representation in terms of a positive Borel measure, $\mu$, supported on an infinite point set, $I \subseteq \mathbb{R}$, such that

$$
u_{n}=\left\langle u, x^{n}\right\rangle=\int_{I} x^{n} d \mu(x), n \geq 0 .
$$

If $\mu$ is an absolutely continuous measure supported on $I$, and $w$ denotes its RadonNikodym derivative with respect to the Lebesgue measure, then we will also say that $\left\{P_{n}\right\}_{n \geq 0}$ is orthogonal with respect to the weight $w$.

Associated with any SMOP there exist sequences $\left\{\gamma_{n}\right\}_{n \geq 0}$ and $\left\{\beta_{n}\right\}_{n \geq 0}$ of positive real numbers and real numbers, respectively, such that the three-term recurrence relation holds [20]:

$$
P_{n+1}(x)=\left(x-\beta_{n}\right) P_{n}(x)-\gamma_{n} P_{n-1}(x), \quad n \geq 1,
$$

with $P_{0}(x)=1$ and $P_{1}(x)=x-\beta_{0}$.

Given a SMOP with respect to $u$, the sequence of associated polynomials of the first kind is defined by

$$
P_{n}^{(1)}(x)=\left\langle u_{[t]}, \frac{P_{n+1}(x)-P_{n+1}(t)}{x-t}\right\rangle, \quad n \geq 0,
$$

as we are assuming $u_{0}=1$. Here, $u_{[t]}$ denotes the action of $u$ on the variable $t$.

The sequence $\left\{P_{n}^{(1)}\right\}_{n \geq 0}$ also satisfies a three-term recurrence relation,

$$
P_{n}^{(1)}(x)=\left(x-\beta_{n}\right) P_{n-1}^{(1)}(x)-\gamma_{n} P_{n-2}^{(1)}(x), \quad n \geq 1,
$$

with $P_{-1}^{(1)}(x)=0$ and $P_{0}^{(1)}(x)=1$.

The Stieltjes function of $u$ is defined by $S(x)=\sum_{n=0}^{\infty} \frac{u_{n}}{x^{n+1}}$. Note that if $u$ is positivedefinite, defined by (4), then $S$ is given by

$$
S(x)=\int_{I} \frac{d \mu(t)}{x-t}, \quad x \in \mathbb{C} \backslash I .
$$

The sequence of functions of the second kind corresponding to $\left\{P_{n}\right\}_{n \geq 0}$ is defined as follows:

$$
q_{n+1}=P_{n+1} S-P_{n}^{(1)}, \quad n \geq 0, q_{0}=S .
$$

Whenever $u$ is positive-definite, defined by (4), the $q_{n}$ 's are given in terms of an integral formula,

$$
q_{n}(x)=\int_{I} \frac{P_{n}(t)}{x-t} d \mu(t), \quad n \geq 0
$$

The Stieltjes function $S$ is said to be Laguerre-Hahn if there exist polynomials $A, B, C, D$, with $A \neq 0$, such that it satisfies a Riccati differential equation [12]

$$
A S^{\prime}=B S^{2}+C S+D
$$


The corresponding sequence of orthogonal polynomials is called Laguerre-Hahn. If $B=0$, then $S$ is said to be Laguerre-Hahn affine or semi-classical.

Note that equation $(7)$ is equivalent to the distributional equation $[12,14]$

$$
\mathcal{D}(A u)=\psi u+B\left(x^{-1} u^{2}\right), \quad \psi=A^{\prime}+C,
$$

being the left product of $u$ by a polynomial defined as

$$
\langle g u, f\rangle=\langle u, g f\rangle, g, f \in \mathbb{P}
$$

the derivative defined as

$$
\langle\mathcal{D} u, f\rangle=-\left\langle u, f^{\prime}\right\rangle, \quad f \in \mathbb{P},
$$

the product of two linear functionals defined as

$$
\langle u v, f\rangle=\langle u, v f\rangle, f \in \mathbb{P},
$$

and the linear functional $x^{-1} u$ defined as

$$
\left\langle x^{-1} u, f\right\rangle=\left\langle u, \Theta_{0} f\right\rangle, \quad \Theta_{0} f(x)=\frac{f(x)-f(0)}{x}, f \in \mathbb{P} .
$$

For further purposes we show the generalization of the above formula, that is, we define $p^{-1} u$, with $p$ a polynomial $p(x)=\prod_{i=1}^{s}\left(x-c_{i}\right)^{m_{i}}$. Let us denote the set of the zeroes of $p$, counting multiplicities, by $\mathcal{R}$. The linear functional $p^{-1} u$ is defined as (see [15])

$$
\left\langle p^{-1}(x) u, f\right\rangle=\left\langle u, \Theta_{\mathcal{R}} f\right\rangle, \quad \Theta_{\mathcal{R}} f(x)=\frac{f(x)-\mathcal{L}_{\mathcal{R}} f(x)}{p(x)}, f \in \mathbb{P},
$$

where $\mathcal{L}_{\mathcal{R}} f$ is the interpolating polynomial of $f, \mathcal{L}_{\mathcal{R}} f(x)=\sum_{i=1}^{s} \sum_{k=1}^{m_{i}} f^{(k-1)}\left(c_{i}\right) \ell_{i, k}(x)$, with $\ell_{i, k}$ the polynomial such that

$$
\ell_{i, k}^{(\mu)}=\left\{\begin{array}{l}
1 \text { if } i=k, \mu=k-1 \\
0 \text { otherwise }
\end{array}\right.
$$

If $u$ is positive-definite, defined in terms of a weight, $w$, then the semi-classical character of $u, \mathcal{D}(A u)=\psi u, \operatorname{deg}(\psi) \geq 1$, is equivalent to $w^{\prime} / w=C / A$ with $w$ satisfying the boundary conditions [12]

$$
\left.x^{n} A(x) w(x)\right|_{a, b}=0, \quad n \geq 0,
$$

where $a, b$ (eventually $a$ and/or $b$ infinite) are linked with the roots of $A$. In such a case, $w$ is the weight function on the support $I=[a, b], \operatorname{thus,} \operatorname{supp}(w)=I$.

We consider the polynomial and rational transformations of linear functionals (see $[17,18,21])$ :

(a) Christoffel transformation

$$
\tilde{u}=(x-c) u, \quad c \notin \operatorname{supp}(u),
$$


the corresponding orthogonal polynomials, $\tilde{P}_{n}$, satisfy

$$
(x-c) \tilde{P}_{n}(x)=P_{n+1}(x)-a_{n} P_{n}(x), \quad a_{n}=P_{n+1}(c) / P_{n}(c) .
$$

(b) Geronimus transformation

$$
\tilde{u}=(x-c)^{-1} u+M \delta(x-c), \quad c \notin \operatorname{supp}(u),
$$

with $M$ a nonzero constant, and $\delta(x-c)$ the Dirac Delta at $c$. The corresponding orthogonal polynomials, $\tilde{P}_{n}$, satisfy

$$
\tilde{P}_{n}(x)=P_{n}(x)-b_{n} P_{n-1}(x), \quad b_{n}=a_{n-1}-\left(\frac{1}{a_{n-1}-r_{n-1}}-M \frac{P_{n-1}^{2}(c)}{\left\langle u, P_{n-1}^{2}\right\rangle}\right)^{-1},
$$

where $a_{n}$ is defined in (9) and $r_{n}=q_{n+1}(c) / q_{n}(c)$.

Throughout the paper we will use the following matrices associated with the SMOP $\left\{P_{n}\right\}_{n \geq 0}$ :

$$
\Psi_{n}=\left[\begin{array}{ll}
P_{n+1} & P_{n}^{(1)}
\end{array}\right]^{T}, \mathcal{Y}_{n}=\left[\begin{array}{cc}
P_{n+1} & q_{n+1} / w \\
P_{n} & q_{n} / w
\end{array}\right], \quad n \geq 0
$$

In the account of (5) and (6), we have the recurrence relations

$$
\begin{aligned}
& \Psi_{n}(x)=\left(x-\beta_{n}\right) \Psi_{n-1}(x)-\gamma_{n} \Psi_{n-2}(x), \quad n \geq 1, \\
& \mathcal{Y}_{n}=\mathcal{A}_{n} \mathcal{Y}_{n-1}, \quad \mathcal{A}_{n}=\left[\begin{array}{cc}
x-\beta_{n}-\gamma_{n} \\
1 & 0
\end{array}\right], \quad n \geq 1,
\end{aligned}
$$

with initial conditions

$$
\Psi_{-1}=\left[\begin{array}{ll}
P_{0} & P_{-1}^{(1)}
\end{array}\right]^{T}, \Psi_{0}=\left[\begin{array}{ll}
P_{1} & P_{0}^{(1)}
\end{array}\right]^{T}, \mathcal{Y}_{0}=\left[\begin{array}{cc}
x-\beta_{0} & 1 \\
1 & 0
\end{array}\right]
$$

As usual, $\mathcal{A}_{n}$ is called the transfer matrix.

The matrices (12) will play a relevant role in the sequel. Indeed, Laguerre-Hahn orthogonal polynomials are characterized in terms of differential systems for $\left\{\Psi_{n}\right\}_{n \geq 0}$ : there holds the equivalence between (7) and

$$
A \Psi_{n}^{\prime}=\mathcal{M}_{n} \Psi_{n}+\mathcal{N}_{n} \Psi_{n-1}, \quad n \geq 0 .
$$

Here, $A$ is the same as in (7) and $\mathcal{M}_{n}, \mathcal{N}_{n}$ are $2 \times 2$ matrices whose entries are bounded degree polynomials depending on the coefficients of the Riccati equation [16, Theorem 1]. In the semi-classical case, that is, $B \equiv 0$ in $(7)$, when dealing with weights, there holds the equivalence between the semi-classical character of $w, w^{\prime} / w=C / A$, and the differential system

$$
A \mathcal{Y}_{n}^{\prime}=\mathcal{B}_{n} \mathcal{Y}_{n}, \quad n \geq 1
$$

Here, $\mathcal{B}_{n}$ is a $2 \times 2$ matrix whose entries are bounded degree polynomials depending on the polynomials $A, C$ (see [22, Theorem 2] and [11]).

Henceforth, $(X)_{(i, j)}$ denotes the $(i, j)$ entry in the matrix $X$, and $I$ denotes the identity matrix. 


\section{The Christoffel-Geronimus transformations: matrix relations}

In this section we will write the matrix modifications for $\left\{\Psi_{n}\right\}_{n \geq 0}$ under the transformations (8) and (10).

\subsection{Christoffel transformation}

Theorem 3.1. Let $\left\{P_{n}\right\}_{n \geq 0}$ be a SMOP and let $\left\{\tilde{P}_{n}\right\}_{n \geq 0}$ be the SMOP related to the modification (8). Denote by $\left\{\Psi_{n}\right\}_{n \geq 0}$ and $\left\{\tilde{\Psi}_{n}\right\}_{n \geq 0}$ the corresponding sequences defined through (12). The following relation holds:

$$
(x-c) \widetilde{\Psi}_{n}(x)=\mathcal{S}_{n}(x ; c) \Psi_{n+1}(x)+\mathcal{T}_{n}(x ; c) \Psi_{n}(x), \quad n \geq 0,
$$

where

$$
\mathcal{S}_{n}(x ; c)=\left[\begin{array}{cc}
1 & 0 \\
-1 & x-c
\end{array}\right], \mathcal{T}_{n}(x ; c)=-a_{n+1} \mathcal{S}_{n}(x ; c)
$$

Proof. Let us denote by $\tilde{P}_{n}^{(1)}$ the associated polynomial of the first kind related to $\tilde{P}_{n}$. Let us first prove the identity

$$
(x-c) \tilde{P}_{n}^{(1)}(x)=a_{n+1} P_{n+1}(x)-P_{n+2}(x)+(x-c)\left(P_{n+1}^{(1)}(x)-a_{n+1} P_{n}^{(1)}(x)\right) .
$$

Using the definition of $\tilde{P}_{n}^{(1)}$ as well as (9), we get, after some basic computations,

$$
(x-c) \tilde{P}_{n}^{(1)}(x)=\left\langle u_{[t]}, \frac{t A_{n}(x)-x A_{n}(t)}{x-t}\right\rangle-c\left\langle u_{[t]}, \frac{A_{n}(x)-A_{n}(t)}{x-t}\right\rangle,
$$

where $A_{n}(x)=P_{n+2}(x)-a_{n+1} P_{n+1}(x)$.

Note that

$$
\left\langle u_{[t]}, \frac{t A_{n}(x)-x A_{n}(t)}{x-t}\right\rangle=\left\langle u_{[t]}, \frac{(t-x) A_{n}(x)-(x-t) A_{n}(t)}{x-t}\right\rangle+\left\langle u_{[t]}, \frac{x A_{n}(x)-t A_{n}(t)}{x-t}\right\rangle,
$$

thus,

$$
\left\langle u_{[t]}, \frac{t A_{n}(x)-x A_{n}(t)}{x-t}\right\rangle=-A_{n}(x)+x P_{n+1}^{(1)}(x)-a_{n+1} x P_{n}^{(1)}(x),
$$

where we have used $\left\langle u_{[t]},\left(-A_{n}(x)-A_{n}(t)\right)\right\rangle=-A_{n}(x)$ together with the recurrence relation (5). Also,

$$
\left\langle u_{[t]}, \frac{A_{n}(x)-A_{n}(t)}{x-t}\right\rangle=P_{n+1}^{(1)}(x)-a_{n+1} P_{n}^{(1)}(x) .
$$

Therefore, (20) and (21) yield (19).

Equation (17) is the matrix form of (9) and (19).

The recurrence relation coefficients for the transformed orthogonal polynomials can also be obtained using the matrix identities (17). Indeed, we recover the results, e.g., in [21]. More information can be obtained in [15, 18, 23]. 
Corollary 3.2. Under the notation of (9), the recurrence coefficients of the SMOP $\left\{\tilde{P}_{n}\right\}_{n \geq 0}$ related to (8) are transformed according to the formulas

$$
\tilde{\beta}_{n}=\beta_{n+1}+a_{n+1}-a_{n}, \quad \tilde{\gamma}_{n}=\gamma_{n} \frac{a_{n}}{a_{n-1}} .
$$

Proof. Multiply the recurrence relation $\widetilde{\Psi}_{n}=\left(x-\tilde{\beta}_{n}\right) \widetilde{\Psi}_{n-1}-\tilde{\gamma}_{n} \widetilde{\Psi}_{n-2}, \quad n \geq 1$, by $(x-c)$ and use (17), thus obtaining

$$
\mathcal{S}_{n} \Psi_{n+1}+\mathcal{T}_{n} \Psi_{n}=\left(x-\tilde{\beta}_{n}\right)\left(\mathcal{S}_{n-1} \Psi_{n}+\mathcal{T}_{n-1} \Psi_{n-1}\right)-\tilde{\gamma}_{n}\left(\mathcal{S}_{n-2} \Psi_{n-1}+\mathcal{T}_{n-2} \Psi_{n-2}\right) .
$$

The use of the recurrence relation (13) in the above equality yields

$$
\mathcal{M}_{n, 1} \Psi_{n}=\mathcal{M}_{n, 2} \Psi_{n-1}, \quad n \geq 0
$$

where

$$
\begin{aligned}
& \mathcal{M}_{n, 1}=\left(x-\beta_{n+1}\right) \mathcal{S}_{n}+\mathcal{T}_{n}-\left(x-\tilde{\beta}_{n}\right) \mathcal{S}_{n-1}-\frac{\tilde{\gamma}_{n}}{\gamma_{n}} \mathcal{T}_{n-2} \\
& \mathcal{M}_{n, 2}=\gamma_{n+1} \mathcal{S}_{n}+\left(x-\tilde{\beta}_{n}\right) \mathcal{T}_{n-1}-\tilde{\gamma}_{n} \mathcal{S}_{n-2}-\frac{\tilde{\gamma}_{n}}{\gamma_{n}}\left(x-\beta_{n}\right) \mathcal{T}_{n-2} .
\end{aligned}
$$

After some computations we conclude that $\mathcal{M}_{n, 1}$ and $\mathcal{M}_{n, 1}$ are null matrices. Therefore, we get

$$
\begin{aligned}
& \left(x-\beta_{n+1}\right) \mathcal{S}_{n}+\mathcal{T}_{n}-\left(x-\tilde{\beta}_{n}\right) \mathcal{S}_{n-1}-\frac{\tilde{\gamma}_{n}}{\gamma_{n}} \mathcal{T}_{n-2}=0, \\
& \gamma_{n+1} \mathcal{S}_{n}+\left(x-\tilde{\beta}_{n}\right) \mathcal{T}_{n-1}-\tilde{\gamma}_{n} \mathcal{S}_{n-2}-\frac{\tilde{\gamma}_{n}}{\gamma_{n}}\left(x-\beta_{n}\right) \mathcal{T}_{n-2}=0 .
\end{aligned}
$$

Equation (23) yields only one non-trivial equation,

$$
\tilde{\beta}_{n}=\beta_{n+1}+a_{n+1}-\frac{\tilde{\gamma}_{n}}{\gamma_{n}} a_{n-1} .
$$

Equation (24) yields only one non-trivial equation,

$$
a_{n}=\frac{\tilde{\gamma}_{n}}{\gamma_{n}} a_{n-1}
$$

Equations (25) and (26) yield (22).

\subsection{Geronimus transformation}

Theorem 3.3. Let $\left\{P_{n}\right\}_{n \geq 0}$ be a SMOP and let $\left\{\tilde{P}_{n}\right\}_{n \geq 0}$ be the SMOP related to the modification (10). Denote by $\left\{\Psi_{n}\right\}_{n \geq 0}$ and $\left\{\widetilde{\Psi}_{n}\right\}_{n \geq 0}$ the corresponding sequences defined through (12). The following relation holds:

$$
(x-c) \widetilde{\Psi}_{n}(x)=\mathcal{S}_{n}(x ; c) \Psi_{n+1}(x)+\mathcal{T}_{n}(x ; c) \Psi_{n}(x), \quad n \geq 0,
$$


where

$$
\begin{aligned}
& \mathcal{S}_{n}(x ; c)=\left[\begin{array}{cc}
1-\frac{\left(c-\tilde{\beta}_{n+1}\right) b_{n+1}}{\gamma_{n+1}}-\frac{\tilde{\gamma}_{n+1}}{\gamma_{n+1}}+\frac{\left(x-\beta_{n}\right) \tilde{\gamma}_{n+1} b_{n}}{\gamma_{n} \gamma_{n+1}} & 0 \\
\frac{b_{n+1}}{\gamma_{n+1}} & \frac{b_{n+1}}{\gamma_{n+1}}
\end{array}\right], \\
& \mathcal{T}_{n}(x ; c)=\left[\begin{array}{cc}
t_{n} & 0 \\
1-\frac{\left(x-\beta_{n+1}\right) b_{n+1}}{\gamma_{n+1}} & 1-\frac{\left(x-\beta_{n+1}\right) b_{n+1}}{\gamma_{n+1}}
\end{array}\right],
\end{aligned}
$$

where

$$
t_{n}=-b_{n+2}+\tilde{\beta}_{n+1}-c+\frac{\tilde{\gamma}_{n+1} b_{n}}{\gamma_{n}}-\left(x-\beta_{n+1}\right)\left(\left(\mathcal{S}_{n}\right)_{(1,1)}-1\right)
$$

Proof. Let us first prove the identity

$$
(x-c) \tilde{P}_{n}^{(1)}(x)=P_{n+1}(x)-b_{n+1} P_{n}(x)+P_{n}^{(1)}(x)-b_{n+1} P_{n-1}^{(1)}(x) .
$$

Note that

$$
(x-c) \tilde{P}_{n}^{(1)}(x)=\left\langle(x-t) \tilde{u}_{[t]}, \frac{\tilde{P}_{n+1}(x)-\tilde{P}_{n+1}(t)}{x-t}\right\rangle+\left\langle(t-c) \tilde{u}_{[t]}, \frac{\tilde{P}_{n+1}(x)-\tilde{P}_{n+1}(t)}{x-t}\right\rangle .
$$

In the account of the orthogonality relation, the first integral in (31) gives us

$$
\left\langle\tilde{u}_{[t]}, \tilde{P}_{n+1}(x)\right\rangle=\tilde{P}_{n+1}(x) .
$$

The second integral in (31) gives us, in the account of (11),

$$
\left\langle u_{[t]}, \frac{\tilde{P}_{n+1}(x)-\tilde{P}_{n+1}(t)}{x-t}\right\rangle=P_{n}^{(1)}(x)-b_{n+1} P_{n-1}^{(1)}(x) .
$$

Therefore, from (32) and (33) we get (30).

Now let us write, in the matrix notation, (11) and (30), that is,

$$
(x-c) \widetilde{\Psi}_{n}=\mathcal{A} \Psi_{n+1}+\mathcal{B}_{n} \Psi_{n}+\mathcal{C}_{n} \Psi_{n-1}+\mathcal{D}_{n} \Psi_{n-2},
$$

with

$$
\begin{gathered}
\mathcal{A}=\left[\begin{array}{ll}
1 & 0 \\
0 & 0
\end{array}\right], \quad \mathcal{B}_{n}=\left[\begin{array}{cc}
\tilde{\beta}_{n+1}-b_{n+2}-c & 0 \\
1 & 1
\end{array}\right], \\
\mathcal{C}_{n}=\left[\begin{array}{cc}
\left(c-\tilde{\beta}_{n+1}\right) b_{n+1}+\tilde{\gamma}_{n+1} & 0 \\
-b_{n+1} & -b_{n+1}
\end{array}\right], \mathcal{D}_{n}=\left[\begin{array}{cc}
-\tilde{\gamma}_{n+1} b_{n} & 0 \\
0 & 0
\end{array}\right] .
\end{gathered}
$$

The use of the recurrence relation (13) yields

$$
(x-c) \widetilde{\Psi}_{n}=\mathcal{S}_{n} \Psi_{n+1}+\mathcal{T}_{n} \Psi_{n},
$$

with

$\mathcal{S}_{n}=\mathcal{A}-\frac{\mathcal{C}_{n}}{\gamma_{n+1}}-\frac{\left(x-\beta_{n}\right)}{\gamma_{n} \gamma_{n+1}} \mathcal{D}_{n}, \quad \mathcal{T}_{n}=\mathcal{B}_{n}+\frac{\left(x-\beta_{n+1}\right)}{\gamma_{n+1}} \mathcal{C}_{n}+\left(\frac{\left(x-\beta_{n}\right)\left(x-\beta_{n+1}\right)}{\gamma_{n} \gamma_{n+1}}-\frac{1}{\gamma_{n}}\right) \mathcal{D}_{n}$. 
Hence, we obtain (27) with the matrices $\mathcal{S}_{n}, \mathcal{T}_{n}$ given in (28) and (29).

Following the same technique as in Corollary 3.2, and using the matrix relations (27), we recover the recurrence coefficients of the modified polynomials (see, e.g., [21]).

Corollary 3.4. Under the notation of (11), the recurrence coefficients of the SMOP $\left\{\tilde{P}_{n}\right\}_{n \geq 0}$ related to (10) are transformed according to the formulas

$$
\tilde{\beta}_{n}=\beta_{n}+b_{n+1}-b_{n}, \quad \tilde{\gamma}_{n}=\gamma_{n-1} \frac{b_{n}}{b_{n-1}} .
$$

\subsection{Iterations - General formulas}

In this section we consider the iteration of (8) and (10). Without loss of generalization, and for simplicity matters, in (35) and (36) we take $c_{i} \neq c_{j}, \nu_{i} \neq \nu_{j}, i \neq j$. We denote the iterated transformation (8) by

$$
u^{[K]}=\prod_{j=1}^{K}\left(x-c_{j}\right) u, \quad c_{j} \notin \operatorname{supp}(u), j=1, \ldots, K,
$$

and the iterated transformation (10) by

$$
u_{[L]}=\frac{1}{\prod_{j=1}^{L}\left(x-\nu_{j}\right)} u+\sum_{j=1}^{L} M_{j} \delta\left(x-\nu_{j}\right), \quad \nu_{j} \notin \operatorname{supp}(u), j=1, \ldots, L
$$

with $M_{j}$ nonzero constants, $j=1, \ldots, L$. The monic orthogonal polynomials related to (35) and (36) will be denoted by $P_{n, K}$, and $P_{n,, L}$, respectively. The corresponding vectors defined in (12) related to $u^{[K]}$ will be denoted by $\Psi_{n, K, \text {, }}$, and the ones related to $u_{[L]}$ will be denoted by $\Psi_{n,, L}$. Recall that the vectors related to $u$ are denoted by $\Psi_{n}$.

As a consequence of Theorems 3.1 and 3.3 we obtain the following result.

Theorem 3.5. For the modification (35), the following relation holds, for all $n \geq 0$ :

$$
\prod_{j=1}^{K}\left(x-c_{j}\right) \Psi_{n, K, \cdot}(x)=\mathcal{S}_{n}\left(x ; c_{k}, \ldots, c_{1}\right) \Psi_{n+1}(x)+\mathcal{T}_{n}\left(x ; c_{k}, \ldots, c_{1}\right) \Psi_{n}(x)
$$

where the matrices $\mathcal{S}_{n}\left(x ; c_{k}, \ldots, c_{1}\right), \mathcal{T}_{n}\left(x ; c_{k}, \ldots, c_{1}\right)$ are defined recursively through

$$
\begin{aligned}
& \mathcal{S}_{n}\left(x ; c_{k}, \ldots, c_{1}\right)=\left(x-\beta_{n+2}\right) \mathcal{S}_{n}\left(x ; c_{k}\right) \mathcal{S}_{n+1}\left(x ; c_{k-1}, \ldots c_{1}\right) \\
&+\mathcal{S}_{n}\left(x ; c_{k}\right) \mathcal{T}_{n+1}\left(x ; c_{k-1}, \ldots, c_{1}\right)+\mathcal{T}_{n}\left(x ; c_{k}\right) \mathcal{S}_{n}\left(x ; c_{k-1}, \ldots, c_{1}\right), \\
& \mathcal{T}_{n}\left(x ; c_{k}, \ldots, c_{1}\right)=-\gamma_{n+2} \mathcal{S}_{n}\left(x ; c_{k}\right) \mathcal{S}_{n+1}\left(x ; c_{k-1}, \ldots, c_{1}\right) \\
&+\mathcal{T}_{n}\left(x ; c_{k}\right) \mathcal{T}_{n}\left(x ; c_{k-1}, \ldots, c_{1}\right),
\end{aligned}
$$

with initial conditions

$$
\mathcal{S}_{n}\left(x ; c_{1}\right)=\left[\begin{array}{cc}
1 & 0 \\
-1 & x-c_{1}
\end{array}\right], \mathcal{T}_{n}\left(x ; c_{1}\right)=-a_{n+1} \mathcal{S}_{n}\left(x ; c_{1}\right)
$$


For the modification (36), the following relation holds, for all $n \geq 0$ :

$$
\prod_{j=1}^{L}\left(x-\nu_{j}\right) \Psi_{n, \cdot, L}(x)=\mathcal{S}_{n}\left(x ; \nu_{L}, \ldots, \nu_{1}\right) \Psi_{n+1}(x)+\mathcal{T}_{n}\left(x ; \nu_{L}, \ldots, \nu_{1}\right) \Psi_{n}(x)
$$

where the matrices $\mathcal{S}_{n}\left(x ; \nu_{L}, \ldots, \nu_{1}\right), \mathcal{T}_{n}\left(x ; \nu_{L}, \ldots, \nu_{1}\right)$ are defined recursively through

$$
\begin{aligned}
& \mathcal{S}_{n}\left(x ; \nu_{L}, \ldots, \nu_{1}\right)=\left(x-\beta_{n+2}\right) \mathcal{S}_{n}\left(x ; \nu_{L}\right) \mathcal{S}_{n+1}\left(x ; \nu_{L-1}, \ldots, \nu_{1}\right) \\
&+\mathcal{S}_{n}\left(x ; \nu_{L}\right) \mathcal{T}_{n+1}\left(x ; \nu_{L-1}, \ldots, \nu_{1}\right)+\mathcal{T}_{n}\left(x ; \nu_{L}\right) \mathcal{S}_{n}\left(x ; \nu_{L-1}, \ldots, \nu_{1}\right), \\
& \mathcal{T}_{n}\left(x ; \nu_{L}, \ldots, \nu_{1}\right)=-\gamma_{n+2} \mathcal{S}_{n}\left(x ; \nu_{L}\right) \mathcal{S}_{n+1}\left(x ; \nu_{L-1}, \ldots, \nu_{1}\right) \\
&+\mathcal{T}_{n}\left(x ; \nu_{L}\right) \mathcal{T}_{n}\left(x ; \nu_{L-1}, \ldots, \nu_{1}\right)
\end{aligned}
$$

with initial conditions

$$
\begin{aligned}
& \mathcal{S}_{n}\left(x ; \nu_{1}\right)=\left[\begin{array}{cc}
1-\frac{\left(\nu_{1}-\tilde{\beta}_{n+1}\right) b_{n+1}}{\gamma_{n+1}}-\frac{\tilde{\gamma}_{n+1}}{\gamma_{n+1}}+\frac{\left(x-\beta_{n}\right) \tilde{\gamma}_{n+1} b_{n}}{\gamma_{n} \gamma_{n+1}} & 0 \\
\frac{b_{n+1}}{\gamma_{n+1}} & \frac{b_{n+1}}{\gamma_{n+1}}
\end{array}\right], \\
& \mathcal{T}_{n}\left(x ; \nu_{1}\right)=\left[\begin{array}{cc}
t_{n} & 0 \\
1-\frac{\left(x-\beta_{n+1}\right) b_{n+1}}{\gamma_{n+1}} & 1-\frac{\left(x-\beta_{n+1}\right) b_{n+1}}{\gamma_{n+1}}
\end{array}\right],
\end{aligned}
$$

where

$$
t_{n}=-b_{n+2}+\tilde{\beta}_{n+1}-\nu_{1}+\frac{\tilde{\gamma}_{n+1} b_{n}}{\gamma_{n}}-\left(x-\beta_{n+1}\right)\left(\left(\mathcal{S}_{n}\left(x ; \nu_{1}\right)\right)_{(1,1)}-1\right) .
$$

Remark 3.6. If we consider multiplicity greater than one of some $c_{j}$ or $\nu_{j}$ in (35) and (36) we get a more general situation. More information on this topic can be obtained in $[1,4,5,14,24]$.

\section{Modifications within the Laguerre-Hahn class}

Recall that the Laguerre-Hahn class is closed under the Christoffel and Geronimus transformations [12]. Let us take a Laguerre-Hahn Stieltjes function, $S$, and let $\tilde{S}$ be its Stieljtes modified under the Christoffel or Geronimus transformations. Recall the equivalence between the Riccati equation for $\tilde{S}$, say

$$
\tilde{A} \tilde{S}^{\prime}=\tilde{B} \tilde{S}^{2}+\tilde{C} \tilde{S}+\tilde{D}
$$

and a structure relation for the corresponding $\widetilde{\Psi}_{n}$,

$$
\tilde{A} \widetilde{\Psi}_{n}^{\prime}=\tilde{\mathcal{M}}_{n} \tilde{\Psi}_{n}+\tilde{\mathcal{N}}_{n} \widetilde{\Psi}_{n-1}, \quad n \geq 0 .
$$

In what follows we construct the difference-differential equation (39) for the transformed $\widetilde{\Psi}_{n}$, based on the matrices from the difference-differential equation (15) for $\Psi_{n}$ related to $S, A \Psi_{n}^{\prime}=\mathcal{M}_{n} \Psi_{n}+\mathcal{N}_{n} \Psi_{n-1}$, as well as on the matrices from the identities (17) and $(27),(x-c) \widetilde{\Psi}_{n}=\mathcal{S}_{n} \Psi_{n+1}+\mathcal{T}_{n} \Psi_{n}$.

Theorem 4.1. Let $\left\{P_{n}\right\}_{n \geq 0}$ be a SMOP related to a Laguerre-Hahn Stieltjes function $S$ satisfying $A S^{\prime}=B S^{2}+C S+D$, equivalently, the corresponding $\left\{\Psi_{n}\right\}_{n \geq 0}$ satisfying the 
difference-differential equation (15),

$$
A \Psi_{n}^{\prime}=\mathcal{M}_{n} \Psi_{n}+\mathcal{N}_{n} \Psi_{n-1}, \quad n \geq 0 .
$$

Let $\left\{\tilde{P}_{n}\right\}_{n \geq 0}$ be the SMOP related to the modified Stieltjes function under the Christoffel or Geronimus transformations (8) or (10), say, $\tilde{S}$, satisfying

$$
\tilde{A} \tilde{S}^{\prime}=\tilde{B} \tilde{S}^{2}+\tilde{C} \tilde{S}+\tilde{D}, \quad \tilde{A}(x)=(x-c) A(x) .
$$

There holds the equivalence between (40) and the difference-differential equation

$$
\tilde{A} \widetilde{\Psi}_{n}^{\prime}=\tilde{\mathcal{M}}_{n} \widetilde{\Psi}_{n}+\tilde{\mathcal{N}}_{n} \widetilde{\Psi}_{n-1}
$$

with the matrices $\tilde{\mathcal{M}}_{n}, \tilde{\mathcal{N}}_{n}$ given by

$$
\tilde{\mathcal{M}}_{n}=\left(\widehat{\mathcal{S}}_{n}+\frac{1}{\gamma_{n+1}} \tilde{\mathcal{N}}_{n} \mathcal{T}_{n-1}\right) \mathcal{S}_{n}^{-1}, \quad \tilde{\mathcal{N}}_{n}=\left(\widehat{\mathcal{T}}_{n} \mathcal{T}_{n}^{-1}-\widehat{\mathcal{S}}_{n} \mathcal{S}_{n}^{-1}\right) \mathcal{V}_{n}^{-1}
$$

where

$$
\begin{gathered}
\widehat{\mathcal{S}}_{n}=(x-c)\left(A \mathcal{S}_{n}^{\prime}+\mathcal{S}_{n} \mathcal{M}_{n+1}-\frac{1}{\gamma_{n+1}} \mathcal{T}_{n} \mathcal{N}_{n}\right)-A \mathcal{S}_{n} \\
\widehat{\mathcal{T}}_{n}=(x-c)\left(A \mathcal{T}_{n}^{\prime}+\mathcal{S}_{n} \mathcal{N}_{n+1}+\mathcal{T}_{n}\left(\mathcal{M}_{n}+\frac{\left(x-\beta_{n+1}\right)}{\gamma_{n+1}} \mathcal{N}_{n}\right)\right)-A \mathcal{T}_{n}, \\
\mathcal{V}_{n}=\frac{1}{\gamma_{n+1}} \mathcal{T}_{n-1} \mathcal{S}_{n}^{-1}+\left(\mathcal{S}_{n-1}+\frac{\left(x-\beta_{n+1}\right)}{\gamma_{n+1}} \mathcal{T}_{n-1}\right) \mathcal{T}_{n}^{-1}
\end{gathered}
$$

with $\mathcal{S}_{n}, \mathcal{T}_{n}$ the matrices from (17) and (27).

Proof. Set the relations (17) and (27) written as

$$
(x-c) \widetilde{\Psi}_{n}=\mathcal{S}_{n} \Psi_{n+1}+\mathcal{T}_{n} \Psi_{n} .
$$

Take derivatives in (46), multiply by the polynomial $A$, and use (15), thus obtaining

$$
A \widetilde{\Psi}_{n}+A(x-c) \widetilde{\Psi}_{n}^{\prime}=\mathcal{S}_{n, 1} \Psi_{n+1}+\mathcal{T}_{n, 1} \Psi_{n}
$$

with

$\mathcal{S}_{n, 1}=A \mathcal{S}_{n}^{\prime}+\mathcal{S}_{n} \mathcal{M}_{n+1}-\frac{1}{\gamma_{n+1}} \mathcal{T}_{n} \mathcal{N}_{n}, \quad \mathcal{T}_{n, 1}=A \mathcal{T}_{n}^{\prime}+\mathcal{S}_{n} \mathcal{N}_{n+1}+\mathcal{T}_{n}\left(\mathcal{M}_{n}+\frac{\left(x-\beta_{n+1}\right)}{\gamma_{n+1}} \mathcal{N}_{n}\right)$

Multiply (47) by $(x-c)$ and use again (46), thus obtaining

$$
\hat{A} \widetilde{\Psi}_{n}^{\prime}=\widehat{\mathcal{S}}_{n} \Psi_{n+1}+\widehat{\mathcal{T}}_{n} \Psi_{n}
$$

with

$$
\hat{A}=(x-c)^{2} A, \widehat{\mathcal{S}}_{n}=(x-c) \mathcal{S}_{n, 1}-A \mathcal{S}_{n}, \widehat{\mathcal{T}}_{n}=(x-c) \mathcal{T}_{n, 1}-A \mathcal{T}_{n}
$$


To deduce the matrices $\tilde{\mathcal{M}}_{n}, \tilde{\mathcal{N}}_{n}$, in (41) we multiply $(x-c) A \widetilde{\Psi}_{n}^{\prime}=\tilde{\mathcal{M}}_{n} \widetilde{\Psi}_{n}+\tilde{\mathcal{N}}_{n} \widetilde{\Psi}_{n-1}$ by $x-c$ and use (46) and (48), as well as the recurrence relation (13), thus getting

$$
\mathcal{M}_{n, 1} \Psi_{n+1}=\mathcal{M}_{n, 2} \Psi_{n}
$$

with

$\mathcal{M}_{n, 1}=\widehat{\mathcal{S}}_{n}-\tilde{\mathcal{M}}_{n} \mathcal{S}_{n}+\frac{1}{\gamma_{n+1}} \tilde{\mathcal{N}}_{n} \mathcal{T}_{n-1}, \quad \mathcal{M}_{n, 2}=-\widehat{\mathcal{T}}_{n}+\tilde{\mathcal{M}}_{n} \mathcal{T}_{n}+\tilde{\mathcal{N}}_{n} \mathcal{S}_{n-1}+\frac{\left(x-\beta_{n+1}\right)}{\gamma_{n+1}} \tilde{\mathcal{N}}_{n} \mathcal{T}_{n-1}$

Taking into account the structure of the matrices defining $\mathcal{M}_{n, 1}$ and $\mathcal{M}_{n, 2}$, we conclude that $\mathcal{M}_{n, 1}$ and $\mathcal{M}_{n, 2}$ are null matrices. Hence, we obtain that $\tilde{\mathcal{M}}_{n}, \tilde{\mathcal{N}}_{n}$ are given by (42).

\subsection{The semi-classical class: the Christoffel and Geronimus weight transformations}

Recall that the semi-classical class is closed under the Christoffel and Geronimus transformations [12]. Whenever dealing with weights, there holds the equivalence between the semi-classical character of $w$, say $w^{\prime} / w=C / A$, and the differential system (16) for the corresponding $\mathcal{Y}_{n}$,

$$
A \mathcal{Y}_{n}^{\prime}=\mathcal{B}_{n} \mathcal{Y}_{n}, \quad n \geq 1
$$

where $\mathcal{B}_{n}$ is a matrix of polynomial entries [22, Theorem 2].

Let us now consider $\tilde{w}$, a modification of $w$ under the Christoffel or Geronimus transformation. As $\tilde{w}$ is semi-classical, there holds a differential system for the corresponding $\tilde{\mathcal{Y}}_{n}=\left[\begin{array}{cc}\tilde{P}_{n+1} & \tilde{q}_{n+1} / \tilde{w} \\ \tilde{P}_{n} & \tilde{q}_{n} / \tilde{w}\end{array}\right]$,

$$
\tilde{A} \tilde{\mathcal{Y}}_{n}^{\prime}=\tilde{\mathcal{B}}_{n} \tilde{\mathcal{Y}}_{n}, \quad n \geq 1 .
$$

In what follows we see how $\tilde{\mathcal{B}}_{n}$ in (49) and the transfer matrices of $\left\{\tilde{\mathcal{Y}}_{n}\right\}_{n>0}$, henceforth denoted by $\tilde{\mathcal{A}}_{n}$, relate to $\mathcal{B}_{n}$ in (16) and to the transfer matrices $\mathcal{A}_{n}$ of $\left\{\mathcal{Y}_{n}\right\}_{n \geq 0}$.

Theorem 4.2. Let $\left\{P_{n}\right\}_{n \geq 0}$ be a SMOP related to a semi-classical weight $w$ with the corresponding $\left\{\mathcal{Y}_{n}\right\}_{n \geq 0}$ satisfying (16),

$$
A \mathcal{Y}_{n}^{\prime}=\mathcal{B}_{n} \mathcal{Y}_{n}, \quad n \geq 1
$$

Let $\mathcal{A}_{n}$ be the transfer matrix of $\mathcal{Y}_{n}$.

a) Christoffel case.

a.1) The sequence $\left\{\tilde{\mathcal{Y}}_{n}\right\}_{n \geq 0}$ related to the modified weight $\tilde{w}(x)=(x-c) w(x)$ satisfies

$$
(x-c) \tilde{\mathcal{Y}}_{n}=\mathcal{C}_{n} \mathcal{Y}_{n}, \quad \mathcal{C}_{n}=\mathcal{A}_{n+1}-\boldsymbol{A}_{n}, \quad n \geq 1,
$$

where $\boldsymbol{A}_{n}=\left[\begin{array}{cc}a_{n+1} & 0 \\ 0 & a_{n}\end{array}\right], a_{n}=P_{n+1}(c) / P_{n}(c)$;

a.2) $\left\{\tilde{\mathcal{Y}}_{n}\right\}_{n \geq 0}$ satisfies a linear system (49), $\tilde{A} \tilde{\mathcal{Y}}_{n}^{\prime}=\tilde{\mathcal{B}}_{n} \tilde{\mathcal{Y}}_{n}, \quad n \geq 1$, where

$$
\tilde{A}=(x-c) A, \quad \tilde{\mathcal{B}}_{n}=\left((x-c)\left(A \mathcal{C}_{n}^{\prime}+\mathcal{C}_{n} \mathcal{B}_{n}\right)-A \mathcal{C}_{n}\right) \mathcal{C}_{n}^{-1}
$$


a.3) The transfer matrices of $\tilde{\mathcal{Y}}_{n}$ and $\mathcal{Y}_{n}$ are related through

$$
\tilde{\mathcal{A}}_{n}=\mathcal{C}_{n} \mathcal{A}_{n} \mathcal{C}_{n-1}^{-1}, \quad n \geq 1
$$

b) Geronimus case.

b.1) The sequence $\left\{\tilde{\mathcal{Y}}_{n}\right\}_{n \geq 0}$ related to the modified weight $\tilde{w}(x)=\frac{w(x)}{(x-c)}$ satisfies

$$
\tilde{\mathcal{Y}}_{n}=\mathcal{G}_{n} \mathcal{Y}_{n}, \quad \mathcal{G}_{n}=\mathcal{A}_{n+1}-\boldsymbol{B}_{n}
$$

where $\boldsymbol{B}_{n}=\left[\begin{array}{cc}b_{n+1} & 0 \\ 0 & b_{n}\end{array}\right], b_{n}=q_{n}(c) / q_{n-1}(c)$;

b.2) $\left\{\tilde{\mathcal{Y}}_{n}\right\}_{n \geq 0}$ satisfies a linear system (49), $\tilde{A} \tilde{\mathcal{Y}}_{n}^{\prime}=\tilde{\mathcal{B}}_{n} \tilde{\mathcal{Y}}_{n}, \quad n \geq 1$, where

$$
\tilde{A}=(x-c) A, \quad \tilde{\mathcal{B}}_{n}=(x-c)\left(A \mathcal{G}_{n}^{\prime}+\mathcal{G}_{n} \mathcal{B}_{n}\right) \mathcal{G}_{n}^{-1}
$$

b.3) The transfer matrices of $\tilde{\mathcal{Y}}_{n}$ and $\mathcal{Y}_{n}$ are related through

$$
\tilde{\mathcal{A}}_{n}=\mathcal{G}_{n} \mathcal{A}_{n} \mathcal{G}_{n-1}^{-1} .
$$

Proof. Christoffel case.

a.1) The sequence of functions of the second kind related to $\tilde{w},\left\{\tilde{q}_{n}\right\}$, satisfies

$$
\tilde{q}_{n}(x)=q_{n+1}(x)-a_{n} q_{n}(x), \quad n \geq 1 .
$$

Thus,

$$
(x-c) \tilde{\mathcal{Y}}_{n}=\mathcal{Y}_{n+1}-\mathbf{A}_{n} \mathcal{Y}_{n}
$$

The use of (14), $\mathcal{Y}_{n+1}=\mathcal{A}_{n+1} \mathcal{Y}_{n}$, in the equation above yields (50).

a.2) Take derivatives in (50), then multiply the resulting equation by $(x-c) A$ and use (50) again, thus obtaining

$$
\left(A \mathcal{C}_{n}+\tilde{\mathcal{B}}_{n} \mathcal{C}_{n}\right) \mathcal{Y}_{n}=(x-c)\left(A \mathcal{C}_{n}^{\prime}+\mathcal{C}_{n} \mathcal{B}_{n}\right) \mathcal{Y}_{n}
$$

Hence, we have the linear system (49) with the data (51).

a.3) Relation (52) follows from the use of the recurrence relation for $\mathcal{Y}_{n}$ as well as for $\tilde{\mathcal{Y}}_{n}$, into (50).

Geronimus case.

b.1) The sequence of functions of the second kind related to $\tilde{w},\left\{\tilde{q}_{n}\right\}$, satisfies

$$
(x-c) \tilde{q}_{n}(x)=q_{n}(x)-b_{n} q_{n-1}(x), \quad n \geq 1 .
$$

Thus,

$$
\tilde{\mathcal{Y}}_{n}=\mathcal{Y}_{n+1}-\mathbf{B}_{n} \mathcal{Y}_{n}
$$

The use of (14), $\mathcal{Y}_{n+1}=\mathcal{A}_{n+1} \mathcal{Y}_{n}$, in the equation above yields (53).

b.2) and b.3) are deduced similarly as a.2) and a.3). 


\section{$4.2 \quad$ Examples}

Let $\left\{P_{n}^{L}\right\}_{n \geq 0},\left\{P_{n}^{J}\right\}_{n \geq 0}$ denote the sequences of monic Laguerre and Jacobi antiassociated polynomials, respectively. These are members of the Laguerre-Hahn families of class zero [25].

The recurrence relation coefficients of $\left\{P_{n}^{L}\right\}_{n \geq 0}$ are

$$
\beta_{n}=2 n+\alpha-1, \gamma_{n+1}=n(n+\alpha), \quad n \geq 1, \beta_{0}=\alpha-1+\lambda, \gamma_{1}=\rho,
$$

with $\alpha, \lambda \in \mathbb{C}, \alpha \neq-n, n \geq 1, \rho \in \mathbb{C} \backslash\{0\}$. The recurrence relation coefficients of $\left\{P_{n}^{J}\right\}_{n \geq 0}$ are

$$
\begin{gathered}
\beta_{n}=\frac{\beta^{2}-\alpha^{2}}{(2 n+\alpha+\beta-2)(2 n+\alpha+\beta)}, \\
\gamma_{n+1}=\frac{4 n(n+\alpha)(n+\beta)(n+\alpha+\beta)}{(2 n+\alpha+\beta-1)(2 n+\alpha+\beta)^{2}(2 n+\alpha+\beta+1)}, \quad n \geq 1
\end{gathered}
$$

and $\beta_{0}=\frac{\beta-\alpha}{\alpha+\beta-2}+\lambda, \gamma_{1}=\rho, \alpha, \beta, \lambda \in \mathbb{C}, \alpha \neq-n, \beta \neq-n, n \geq 1, \alpha+\beta \neq-n, n \geq$ $2, \alpha+\beta \neq 2, \rho \in \mathbb{C} \backslash\{0\}$.

The corresponding Stieltjes functions satisfy (7), $A S^{\prime}=B S^{2}+C S+D$, and the corresponding $\Psi_{n}$ satisfy structure relations (15), $A \Psi_{n}^{\prime}=\mathcal{M}_{n} \Psi_{n}+\mathcal{N}_{n} \Psi_{n-1}$, where (see [22, Lemma 6]):

(i) in the $P_{n}^{L}$ case,

$$
\begin{gathered}
A=x, B=-x^{2}-\left(\lambda-2 \beta_{0}\right) x-\alpha \beta_{0}+\rho, C=x-\alpha, D=0, \\
\mathcal{M}_{n}=\left[\begin{array}{cc}
-x+n+\alpha & -B \\
0 & n
\end{array}\right], \quad \mathcal{N}_{n}=n(n+\alpha) I, \quad n \geq 1 ;
\end{gathered}
$$

(ii) in the $P_{n}^{J}$ case,

$$
\begin{aligned}
& A=1-x^{2}, B=(-\alpha-\beta+1) x^{2}+\left(\beta_{0}(\alpha+\beta)+\beta-\alpha\right) x+(\alpha+\beta+1) \rho+\beta_{0}(\alpha-\beta)-1, \\
& C=(\alpha+\beta) x+\alpha-\beta, D=0, \\
& \mathcal{M}_{n}=\left[\begin{array}{cc}
-(\alpha+\beta+n) x-(\alpha-\beta)-\alpha_{n} & -B \\
0 & -n x-\alpha_{n}
\end{array}\right], \quad \mathcal{N}_{n}=\left(-\alpha_{n}^{2}+2 \nu_{n}+n\right) I, \quad n \geq 1,
\end{aligned}
$$

where $\alpha_{n}=\sum_{k=1}^{n} \beta_{k}, \nu_{n}=\sum_{1 \leq i<j \leq n}^{n} \beta_{i} \beta_{j}-\sum_{k=2}^{n} \gamma_{k}, n \geq 1$.

Under the Christoffel transformation (8), the modified Stieltjes function $\tilde{S}$ is given by $\tilde{S}(x)=(x-c) S(x)-1$. Thus, we have $\tilde{A} \tilde{S}^{\prime}=\tilde{B} \tilde{S}^{2}+\tilde{C} \tilde{S}+\tilde{D}$, with

$\tilde{A}(x)=(x-c) A, \tilde{B}=B, \tilde{C}=A+2 B+(x-c) C, \tilde{D}=A+B+(x-c) C+(x-c)^{2} D$.

The difference-differential equation (41) holds with the matrices $\tilde{\mathcal{M}}_{n}, \tilde{\mathcal{N}}_{n}$ given by (42), with the $\mathcal{S}_{n}, \mathcal{T}_{n}$ given in (18).

Under the Geronimus transformation (10), the modified Stieltjes function $\tilde{S}$ is given 


$$
\begin{aligned}
& \text { by } \tilde{S}(x)=\frac{S(x)-S(c)+M}{x-c} \text {. Thus, we have } \tilde{A} \tilde{S}^{\prime}=\tilde{B} \tilde{S}^{2}+\tilde{C} \tilde{S}+\tilde{D} \text {, with } \\
& \begin{array}{r}
\tilde{A}(x)=(x-c) A, \tilde{B}=(x-c)^{2} B, \tilde{C}=-A+2(S(c)-M)(x-c) B+(x-c) C \\
\tilde{D}=(S(c)-M)^{2} B+(S(c)-M) C+D .
\end{array}
\end{aligned}
$$

The difference-differential equation (41) holds with the matrices $\tilde{\mathcal{M}}_{n}, \tilde{\mathcal{N}}_{n}$ given by (42), with the $\mathcal{S}_{n}, \mathcal{T}_{n}$ given in (28)-(29).

\section{Acknowledgements}

The authors are grateful to the anonymous referees for their valuable comments and suggestions.

KC is supported by the Portuguese Government through the Fundação para a Ciência e a Tecnologia (FCT) under the grant SFRH/BPD/101139/2014. This work is partially supported by the Centre for Mathematics of the University of Coimbra UID/MAT/00324/2013, funded by the Portuguese Government through FCT/MCTES and co-funded by the European Regional Development Fund through the Partnership Agreement PT2020.

\section{References}

[1] Christoffel EB. Über die Gaussische Quadratur und eine Verallgemeinerung derselben. J. Reine Angew. Math. 1858;55:61-82.

[2] Geronimus Ya L. On the polynomials orthogonal with respect to a given number sequence. Zap. Mat. Otdel. Khar'kov. Univers. i Nil Mat. i Mehan. 1940;17:3-18.

[3] Geronimus Ya L. On the polynomials orthogonal with respect to a given number sequence and a theorem by W. Hahn. lzv. Akad. Nauk SSSR. 1940;4:215-228.

[4] Uvarov VB. Relation between polynomials orthogonal with different weights. Dokl. Akad. Nauk SSSR. 1959;126:33-36.

[5] Uvarov VB. The connection between systems of polynomials that are orthogonal with respect to different distribution functions. USSR Comput. Math. Math. Phys. 1969;9:25-36.

[6] Bueno MI, Marcellán F. Darboux transformation and perturbation of linear functionals. Linear Algebra Appl. 2004;384:215-242.

[7] Ismail MEH. Classical and Quantum Orthogonal Polynomials in One Variable. vol. 98 of Encyclopedia of Mathematics and its Applications, Cambridge University Press, Cambridge; 2005.

[8] Spiridonov V, Vinet L, Zhedanov A. Spectral transformations, self-similar reductions and orthogonal polynomials. J. Phys. A 1997;30:7621-7637.

[9] Spiridonov V, Vinet L, Zhedanov A. Difference Schrödinger operators with linear and exponential discrete spectra Lett. Math. Phys. 1993;29:63-73.

[10] Witte NS. Bi-orthogonal systems on the unit circle, regular semi-classical weights and integrable systems - II. J. Approx. Theory. 2009;161:565-616.

[11] Magnus AP. Painlevé-type differential equations for the recurrence coefficients of semiclassical orthogonal polynomials. J. Comput. Appl. Math. 1995;57:215-237.

[12] Maroni P. Une théorie algébrique des polynômes orthogonaux. Application aux polynômes orthogonaux semi-classiques. In: Brezinski C, Gori L, Ronveaux A, editors. Orthogonal polynomials and their applications, Vol. 9. Baltzer, Basel: IMACS Ann. Comput. Appl.Math.; 1991. p. 95-130.

[13] Dehesa JS, Marcellán F, Ronveaux A. On orthogonal polynomials with perturbed recurrence relations. J Comput Appl Math. 1990;30:203212. 
[14] Dini J. Sur les formes linéaires et les polynômes orthogonaux de Laguerre-Hahn [doctoral dissertation]. Paris (France): University Pierre et Marie Curie; 1988.

[15] Dini J, Maroni P. La multiplication d'une forme linéaire par une fractton rationnelle. Application aux formes de Laguerre-Hahn. Ann. Polon. Math. 1990;52:175-185.

[16] Branquinho A, Foulquié Moreno A, Paiva A, Rebocho MN. Second-order differential equations in the Laguerre-Hahn class. Appl. Numer. Math. 2015;94:16-32.

[17] Marcellán F, Prianes E. Perturbations of Laguerre-Hahn linear functionals. J. Comput. Appl. Math. 1999;105:109-128.

[18] P. Maroni, Sur la suite de polynômes orthogonaux associée à la forme $u=\delta_{c}+\lambda(x-c)^{-1} L$. Period. Math. Hungar. 1990;21:223-248.

[19] Chihara TS. An introduction to orthogonal polynomials. New York (NY): Gordon and Breach; 1978.

[20] G Szegö. Orthogonal polynomials. 4th ed. Providence Rhode Island: Amer Math Soc Colloq Publ.; 1975.

[21] Zhedanov A. Rational spectral transformations and orthogonal polynomials. J. Comput. Appl. Math. 1997;85:67-86.

[22] Branquinho A, Paiva A, Rebocho MN. Sylvester equations for Laguerre-Hahn orthogonal polynomials on the real line, Appl. Math. Comput. 2013;219:9118-9131.

[23] Gautschi W. An algorithmic implementation of the generalized Christoffel Theorem. In: G. Hämmerlin, editor. Numerical Integration, ISNM 57. Birkhäuser Verlag, Basel; 1982. p. 89-106.

[24] Paszkowski S. Sur des transformations d'une function poinds. In: Brezinski C, Draux A, Magnus AP, Maroni P, Ronveaux A, editors. Polynômes Orthogonaux et Applications. ISNM 1171. Springer Verlag, Berlin; 1985. p. 239-246.

[25] Bouakkaz H, Maroni P. Description des polynômes orthogonaux de Laguerre-Hahn de classe zéro. In: Brezinski C, Gori L, Ronveaux A, editors. Orthogonal polynomials and their applications, Vol. 9. Baltzer, Basel: IMACS Ann. Comput. Appl. Math.; 1991. p. 189-194. 